\title{
Fiordos de Chile: Potencial zona de crianza de Bathylagichthys parini (Pisces: Osmeriformes: Bathylagidae)
}

\author{
Chilean fjords: \\ Potential nursery ground of Bathylagichthys parini (Pisces: Osmeriformes: Bathylagidae) \\ Mauricio F. Landaeta ${ }^{1}$, Jorge Contreras ${ }^{1}$ y Claudia A. Bustos ${ }^{1,2}$
}

\begin{abstract}
${ }^{1}$ Laboratorio de Ictioplancton (LABITI), Facultad de Ciencias del Mar y de Recursos Naturales, Universidad de Valparaíso, Avenida Borgoño 16344, Reñaca, Viña del Mar, Chile. mauricio.landaeta@uv.cl

${ }^{2}$ Programa de Doctorado en Acuicultura, Universidad Católica del Norte, Larrondo 1281, Coquimbo, Chile
\end{abstract}

\begin{abstract}
Eighteen juveniles of Bathylagichthys parini (Osmeriformes: Bathylagidae) were collected in fjords of southern Chile (ca. $48^{\circ} \mathrm{S}-74^{\circ} \mathrm{W}$ ) in November 2008. Standard length and wet weight ranged from 22.6 to $37.7 \mathrm{~mm}$, and between 0.049 and $0.243 \mathrm{~g}$, respectively; density ranged between 3.56 and 41.77 juveniles $x 10 \mathrm{~m}^{2}$. Stomach contents included calanoid copepods (mainly Acartia tonsa and Paracalanus parvus adults and copepodids), ostracods and mussel larvae. This finding suggests that juveniles feed near surface waters and aids evidence to the hypothesis the fj ords are utilized as nursery zone for this rare oceanic species from South Pacific.
\end{abstract}

Key words: Nursery area, Patagonia, food, otoliths

\section{INTRODUCCIÓN}

Los peces de la familia Bathylagidae son pequeños peces marinos (hasta $25 \mathrm{~cm}$ ) que habitan desde aguas superficiales a profundas en todos los océanos (Pequeño \& Matallanas 2003, Cornejo \& Koppelmann 2006, Nelson 2006). Incluye 23 especies distribuidas en ocho géneros. Entre ellos, el género Bathylagichthys que se caracteriza por un sistema de canales dérmicos en la zona del tronco, dientes con múltiples coronas sobre el dentario, la presencia de un mesocoracoide y un postcleitro tripartito en la cintura pectoral y de un urodermo en el esqueleto de la aleta caudal (Kobylyansky 1990). Contiene cinco especies: B. greyae (Cohen, 1958), B. longipinnis (Kobyliansky, 1985), B. problematicus (Lloris \& Rucabado, 1985), B. parini Kobyliansky, 1990 y B. australis Kobyliansky, 1990.

Se han descrito las larvas de Bathylagichthys para algunas especies (John \& Zelck 2001, Uribe \& Balbontín 2005); sin embargo, es escasa la información sobre los estados juveniles (ejemplares con radios de las aletas completas y comienzo de la escamación hasta que el pez ingresa a la población adulta o alcanza la madurez sexual, Kendall et al. 1985). Hasta la fecha, existe solo la breve descripción de un individuo de B. parini (Uribe \& Balbontín 2005) y seis individuos de B. greyae (John \& Zelck 2001). Utilizando 18 juveniles de $B$. parini, recolectados en la zona de fiordos del sur de Chile durante noviembre de 2008, se describen algunas características morfométricas, merísticas, contenido estomacal junto con una breve descripción de los otolitos sagitta de esta especie, discutiendo la hipótesis que esta área es una zona de crianza para esta especie.

\section{Materiales y MÉtodos}

Los ejemplares fueron recolectados durante un crucero oceanográfico llevado a cabo en el sur de Chile (4648'$50^{\circ} 09^{\prime} \mathrm{S}$ ) entre el 1 y 19 de noviembre de 2008, a bordo del buque AGOR ‘Vidal Gormaz’. En cada una de las estaciones oceanográficas (44 en total), se realizaron muestreos de mesozooplancton a través de lances oblicuos con una red Bongo estándar (66 cm de diámetro, $300 \mu \mathrm{m}$ de apertura de malla) equipada con flujómetros TSK (Tsurumi-SeikiKosakusho Co. Ltd., Japón), para estimar el volumen de agua de mar filtrada. Inicialmente, las muestras de plancton fueron fijadas con formalina al 10\% tamponeada con borato de sodio, y posteriormente las larvas y juveniles de peces fueron preservados en etanol al 70\%.

En laboratorio, los individuos de Bathylagichthys parini fueron separados de las muestras analizadas, e identificados según Kobyliansky (1990), Pequeño \& Matallanas (2003) y Uribe \& Balbontín (2005). La densidad fue estandarizada 
a individuos por $10 \mathrm{~m}^{2}$ de acuerdo al volumen filtrado en cada estación positiva. Los peces fueron pesados individualmente usando una balanza Precisa 505M-2020CDRSCS. Cada individuo fue medido utilizando una lupa estereomicroscópica (Leitz Wetzlar, Alemania) con una reglilla en el ocular. Las partes y mediciones corporales mencionadas en el texto están definidas en Matallanas (1986) y Moser (1996). Se realizaron 16 mediciones morfométricas en cada individuo: longitud total (LT), longitud estándar (LE), longitud de la cabeza (LC), diámetro horizontal del ojo (DO), longitud del hocico (LH), longitud predorsal (Lpd), longitud postdorsal (Ld), longitud preanal (Lpa), longitud postanal (La), longitud prepectoral (Lpec), longitud prepélvica (Lpel), longitud de la base de aleta anal (Laa), longitud de la base de aleta dorsal (Lad), distancia entre dorsal y anal (D-A), distancia entre pectoral y ventral $(\mathrm{P}-\mathrm{V})$ y altura del cuerpo (AC).

También se realizaron recuentos de radios dorsales, anales y pélvicos de cada individuo. Se compararon las proporciones con la longitud estándar de cuatro medidas morfométricas (LC, DO, AC y Lpa) con los datos disponibles de trabajos publicados para larvas y adultos de la especie. Se realizaron regresiones lineales por mínimos cuadrados para estudiar las variaciones de estas proporciones en Bathylagichthys parini a lo largo de su ontogenia. Se depositaron dos individuos (25,49 y 29,89 mm LE) en el Museo Nacional de Historia Natural (MNHNC) (números de catálogo MNHNC P7430 y P7431).

Se analizó el contenido estomacal de 16 de los 18 individuos. Para diseccionar el estómago, se utilizaron agujas entomológicas para remover los contenidos. Cada ítem presa fue identificado hasta el nivel taxonómico más bajo posible. Las dimensiones de cada ítem presa fueron medidas con una precisión de 0,01 mm utilizando una reglilla ajustada a un microscopio. El volumen de cada presa fue estimado usando la forma tridimensional que más se pareciera a la presa (Cass-Calay 2003). Para cada ítem presa, se calculó de acuerdo a Hyslop (1980) el porcentaje numérico $(\% \mathrm{~N})$, porcentaje volumétrico $(\% \mathrm{~V})$ y frecuencia de ocurrencia (\%FO) en todos los juveniles que presentaron contenido estomacal. Para posibilitar comparaciones entre ítems presa, el índice de importancia porcentual (\%IIR) de cada presa fue calculado según Cortes (1997).

La descripción de los otolitos sagitta se hizo según Iizuka \& Katayama (2008). Los otolitos fueron extraídos bajo estereomicroscopio con agujas entomológicas, y fotografiados con una cámara Motic Moticam 2000 de 2 MPixel de resolución fijada al microscopio y conectada a un computador. Se midió el radio mayor, perímetro y área de los otolitos utilizando el programa Motic Images Plus $2.0 ®$.

\section{Resultados Y DiscusióN}

Los individuos fueron recolectados solo en el 9,1\% de todas las estaciones oceanográficas, tanto de día como de noche, y se encontraron en una restringida zona dentro de los fiordos y canales cercanos al río Baker, XI Región de Chile, particularmente en el fiordo Steffen $\left(47^{\circ} 45^{\prime} \mathrm{S}, 73^{\circ} 41^{\prime} \mathrm{W}\right)$,

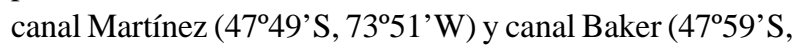
$73^{\circ} 46^{\prime} \mathrm{W}$ ), a pesar que el muestreo de plancton cubrió casi 3 grados de latitud (desde $46^{\circ} 48^{\prime} \mathrm{S}$ hasta $50^{\circ} 09^{\prime} \mathrm{S}$ ). Los juveniles fueron recolectados en aguas con temperatura entre 8,1 y $10,5^{\circ} \mathrm{C}, 4,7$ y 33,9 ups y 6,4 y $7,9 \mathrm{~mL} \mathrm{~L}^{-1}$ de oxígeno disuelto. La densidad estandarizada varió entre 3,56 y 41,77 ind. $10 \mathrm{~m}^{2}$. El peso húmedo de los peces varió entre 0,049 y $0,243 \mathrm{~g}$, con un valor promedio ( \pm una desviación estándar) de 0,115 \pm 0,056 g (Tabla 1). Morfológicamente, los juveniles no tienen el peculiar tejido coroide, situado bajo el ojo en forma de gota de agua, característico de los estados larvales (Uribe \& Balbontín 2005). El cuerpo es alargado y

Tabla 1. Morfometría, recuento de radios de las aletas y peso de juveniles de Bathylagichthys parini en fiordos del sur de Chile. DE = una desviación estándar. Abreviaciones explicadas en el texto / Morphometric, fin ray counts and weight of juvenile Bathylagichthys parini from fjords of southern Chile. $D E=$ one standard deviation. Abbreviations explained in the text

\begin{tabular}{crrrr}
\hline & Promedio & DE & Mínimo & Máximo \\
\hline Morfometría (mm) & & & & \\
LT & 30,824 & 4,877 & 23,094 & 42,991 \\
LE & 27,321 & 4,127 & 22,587 & 37,696 \\
LC & 7,685 & 1,236 & 5,999 & 10,196 \\
DO & 2,161 & 0,512 & 1,675 & 3,788 \\
AC & 4,291 & 0,543 & 3,197 & 5,097 \\
Lpd & 13,737 & 2,475 & 10,787 & 18,897 \\
Ld & 13,676 & 2,091 & 10,393 & 19,094 \\
Lpa & 21,579 & 3,481 & 16,291 & 29,800 \\
La & 5,736 & 1,031 & 4,097 & 7,999 \\
Lpec & 7,973 & 1,252 & 6,196 & 10,800 \\
Lpel & 15,006 & 2,683 & 11,096 & 20,996 \\
Laa & 2,789 & 0,354 & 2,197 & 3,197 \\
Lad & 3,230 & 0,516 & 2,099 & 4,097 \\
LH & 2,286 & 1,252 & 1,00 & 6,940 \\
D-A & 10,455 & 1,830 & 7,097 & 13,490 \\
P-V & 7,344 & 1,572 & 5,294 & 11,588 \\
Recuento de radios & & & & \\
Aleta dorsal & & & 10 & 14 \\
Aleta anal & & & 10 & 14 \\
Aleta pélvica & & & 10 & 12 \\
Peso (g) & 0,115 & 0,056 & 0,049 & 0,243 \\
\hline
\end{tabular}


se adelgaza progresivamente hacia la zona caudal (Fig. 1a); la cabeza es grande, $25,0-29,3 \%$ de LE, el ojo es grande, 6,7-10,0\% de LE. La mandíbula superior se extiende hasta el margen anterior de la órbita del ojo. La aleta dorsal está localizada a la altura de la aleta pélvica, su origen comienza aproximadamente cerca del 50\% de LE (Tabla 1); del mismo modo, la aleta anal se encuentra a la altura de la aleta adiposa dorsal. La longitud preanal es larga, extendiéndose entre 64,4 y 85,5\% de LE. La pigmentación se concentra principalmente en la parte superior del cuerpo, como melanóforos estrellados; además, se observaron parches de melanóforos bajo las cápsulas óticas, sobre el preopérculo, opérculo, narina, mandíbula superior, y en la base de la aleta adiposa (Fig. 1a). El área desde el preopérculo hasta el ano en la región del estómago es mucho más oscura que el resto del cuerpo.

Las proporciones morfométricas seleccionadas se mantuvieron a lo largo de los estados ontogénicos (larvas, juveniles y adultos), observada por una alta bondad de ajuste de las relaciones (valores de $R^{2}$ de las regresiones lineales fueron entre 0,951 y 0,996). Existe un leve incremento en la proporción LC/LE y DO/LE desde el estado larval al juvenil. A su vez, las larvas de Bathylagichthys parini fueron proporcionalmente más altas que los juveniles. Por otro lado, la proporción Lpa/LE se mantuvo relativamente sin cambios a lo largo del desarrollo de la especie.

Dieciséis de los dieciocho estómagos fueron examinados y todos ellos tuvieron contenido. Veinte ítems presa fueron identificados en los contenidos estomacales. Los copépodos (adultos, copepoditos, nauplii y huevos), ostracoda, moluscos y anfípodos fueron los taxa más importantes (Tabla 2). El taxón más importante fue el estado adulto del copépodo eurihalino Acartia tonsa (\%IIR=75,8\%, Tabla 2), que fue ingerido entre 1 y 104 individuos por estómago, y se encontró en todos los individuos ( $\% \mathrm{FO}=100 \%$, Tabla 2$)$. Las presas de pequeño tamaño dominaron en los contenidos estomacales, variando desde $0,004 \mathrm{~mm}^{3}$ (huevos de copépodo) a $0,863 \mathrm{~mm}^{3}$ (anfípodo hyperiidae juvenil), con un promedio de 0,102 \pm 0,242 $\mathrm{mm}^{3}$ (desviación estándar, DE).

Los otolitos sagitta de los juveniles de Bathylagichthys parini son del tipo elíptico (Fig. 1b), bastante similares a los de Leuroglossus schmidti (Iizuka \& Katayama 2008). El radio de los otolitos (promedio \pm DE) fue de 50,99 \pm 6,28 $\mu \mathrm{m}$ (rango: 41,7 a 62,9 $\mu \mathrm{m}$ ), el perímetro de 234,14 \pm 29,22 $\mu \mathrm{m}$ (200,8 a 282,6 $\mu \mathrm{m})$, y el área de 2955,02 \pm 648,52 $\mu \mathrm{m}^{2}\left(2277,7\right.$ a $\left.4053,5 \mu \mathrm{m}^{2}\right)$.

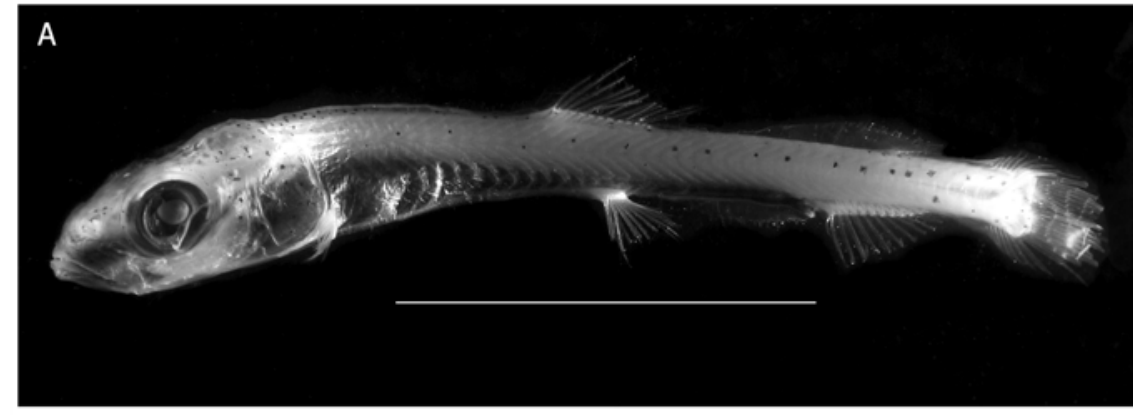

B

Cara externa

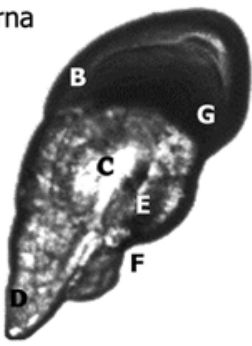

Cara interna

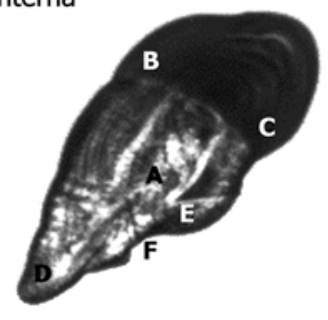

Figura 1. a) Fotografía de Bathylagichthys parini juvenil de $25,49 \mathrm{~mm}$ LE recolectado con red Bongo en los fiordos del sur de Chile. La línea corresponde a $\mathbf{1 0}$ $\mathrm{mm}$. Fotografía de MSc. Iván Hinojosa; b) Morfología del otolito sagitta de juveniles de Bathylagichthys parini. A: sulcus, B: cresta, C: core, D: rostrum, E: antirostrum, F: excisura major, G: domo dorsal / a) Photograph of Bathylagichthys parini juvenile of $25.49 \mathrm{~mm}$ SL collected with Bongo net in fjords of southern Chile. The line corresponds to $10 \mathrm{~mm}$. Photograph by MSc. Iván Hinoj osa; b) Otolith morphology of Bathylagichthys parini juveniles. A: sulcus, B: crist, C: core, D: rostrum, E: antirostrum, $F$ : excisura major, G: dorsal dome 
Tabla 2. Ítems alimenticios identificados en los contenidos estomacales de juveniles de Bathylagichthys parini de fiordos del sur de Chile. $\% \mathrm{~N}=$ porcentaje numérico, $\% \mathrm{~V}=$ porcentaje volumétrico, $\% \mathrm{FO}=$ porcentaje de frecuencia de ocurrencia, \%IIR = índice de importancia relativa porcentual $/$ Food items identified in the gut contents of Bathylagichthys parini juvenile from fjords of southern Chile. $\% \mathrm{~N}=$ numeric percentage, $\% \mathrm{~V}=$ volumetric percentage, \% $\% \mathrm{~F}=$ occurrence frequency percentage, $\% \mathrm{IR}=$ index of relative importance percentage

\begin{tabular}{lrrrr}
\hline Taxa & $\% \mathrm{~N}$ & $\% \mathrm{~V}$ & $\% \mathrm{FO}$ & $\% \mathrm{IIR}$ \\
\hline Larvas de Mytilus chilensis & 2,68 & 1,63 & 18,75 & 0,486 \\
Ostracoda & 3,73 & 15,68 & 56,25 & 6,560 \\
Huevos de copépodo & 0,35 & 0,05 & 18,75 & 0,045 \\
Nauplii de copépodo & 0,12 & 0,04 & 6,25 & 0,006 \\
Copepoditos de Acartia tonsa & 14,47 & 5,38 & 68,75 & 8,200 \\
Copepoditos de Paracalanus parvus & 2,57 & 0,71 & 50,0 & 0,985 \\
Adultos de Acartia tonsa & 67,09 & 59,14 & 100 & 75,845 \\
Adultos de Paracalanus parvus & 4,55 & 8,02 & 81,25 & 6,137 \\
Adultos de Rhincalamus nasutus & 1,28 & 0,70 & 25,0 & 0,297 \\
Adultos de Oithona sp. & 2,68 & 2,22 & 31,25 & 0,920 \\
Harpacticoidea & 0,23 & 0,26 & 12,5 & 0,037 \\
Anfipodo Hyperiidae juvenil & 0,23 & 6,15 & 12,5 & 0,479 \\
Taxa no identificados & 0,02 & 0,02 & 12,5 & 0,003 \\
\hline
\end{tabular}

El registro de varios juveniles de Bathylagichthys parini dentro de fiordos y canales del sur de Chile confirmaría la hipótesis de que esta especie utiliza el área como zona de crianza, sugerida por Pequeño \& Matallanas (2003). Previamente, han sido recolectados estados larvales de esta especie en baja abundancia ( 13 ind. $10 \mathrm{~m}^{2}$ ) principalmente en primavera a lo largo del sur de Chile $\left(41^{\circ} 30^{\prime}-50^{\circ} \mathrm{S}\right)$ (Balbontín \& Bernal 1997, 2005, Bernal \& Balbontín 1999, Uribe \& Balbontín 2005, Bustos et al. 2008). También, los adultos han sido recolectados en la misma área de este estudio (Pequeño \& Matallanas 2003).

La morfología de los juveniles se asemeja a los estados larvales (Uribe \& Balbontín 2005), ya que la mayoría de las proporciones morfológicas se mantuvo entre ambos estados, y las diferencias surgieron principalmente por la falta de tejido coroide bajo el ojo y la presencia de masiva pigmentación oscura sobre el área del estómago. También fueron muy similares a los juveniles de Bathylagichthys greyae descritos por John \& Zelck (2001) y Richards (2006).

En relación a la dieta de Bathylagichthys parini, este es el primer esfuerzo por cuantificar la alimentación de esta especie. La preferencia de copépodos calanoideos de pequeño tamaño fue establecida claramente. También, la importancia de adultos y copepoditos de Acartia tonsa y Paracalanus parvus y ostrácodos en el contenido estomacal de $B$. parini sugieren que la alimentación ocurre en aguas superficiales de tipo estuarino, donde estas presas son abundantes (Rosenberg \& Palma 2003, Castro et al. 2007).
Se ha determinado una dieta similar para peces metamorfoseados de Leuroglossus schmidti y Bathylagus ochotensis, siendo los copépodos y ostrácodos las presas más importantes (Gordon et al. 1985). Pequeño \& Matallanas (2003) también encontraron algunos estados de desarrollo de crustáceos decápodos en un espécimen adulto recolectado en fiordos chilenos durante noviembre de 2006. La potencial distribución superficial de los juveniles de $B$. parini sugerida por la dieta, contrasta con el rango amplio de distribución de juveniles de $B$. greyae, recolectados a diferentes estratos de profundidad (0-80 y 600-800 m de profundidad) en aguas tropicales y subtropicales del Atlántico (John \& Zelck 2001).

La presente descripción de los otolitos sagitta puede ayudar a la identificación correcta de esta especie en el contenido estomacal de peces de importancia comercial, como la merluza austral Merluccius australis y merluza de cola Macruronus magellanicus que podrían predar sobre individuos adultos y juveniles dentro y fuera de los fiordos del sur de Chile.

Aún se ignora cómo las condiciones ambientales de la columna de agua (e.g., baja temperatura y salinidad, Bustos et al. 2008) pueden afectar los hábitos alimenticios y tasas de crecimiento de larvas y juveniles en un ambiente altamente estratificado, y si los individuos adultos recolectados por Kobylyansky (1990) en aguas abiertas de océano Pacífico suroriental corresponden a la misma población que migra extensamente con propósitos reproductivos a las áreas costeras, o pertenecen a otro stock o población con diferentes tácticas reproductivas y zonas de desove y crianza.

\section{Agradecimientos}

Los autores agradecen a Oscar Gallo y la tripulación del AGOR Vidal Gormaz por la recolección de las muestras, y a J. Francisco Ruiz y Katherine Castillo por la separación de las larvas y juveniles de peces de las muestras de zooplancton. Agradecemos a la profesora Bernardita Campos por su ayuda en la identificación de larvas de mitílidos, y al Prof. Fernando Balbontin por sugerencias a una versión anterior del manuscrito. Esta investigación fue financiada parcialmente por el Comité Oceanográfico Nacional de Chile (CONA Chile) a través del proyecto CF14 08-02 (adjudicado a CAB), y el proyecto Fondecyt 11090020 (adjudicado a MFL). Durante la escritura del trabajo, CAB fue financiada por una beca doctoral CONICYT. También agradecemos al MSc. Iván Hinojosa por su trabajo fotográfico. 


\section{LiterATURA CITADA}

Balbontín F \& R Bernal. 1997. Distribución y abundancia del ictioplancton en la zona austral de Chile. Ciencia y Tecnología del Mar 20: 155-163.

Balbontín F \& R Bernal. 2005. Cambios estacionales en la composición y abundancia del ictioplancton de los canales australes entre el Golfo Corcovado y Golfo Elefantes, Chile. Ciencia y Tecnología del Mar 28: 99-111.

Bernal R \& F Balbontín. 1999. Ictioplancton de los fiordos entre el Golfo de Penas y Estrecho de Magallanes y factores ambientales asociados. Ciencia y Tecnología del Mar 22: 143-154.

Bustos CA, MF Landaeta \& F Balbontín. 2008. Efectos ambientales sobre la variabilidad espacial del ictioplancton de Chile austral durante noviembre de 2005. Revista Chilena de Historia Natural 81: 205-219.

Cass-Calay SL. 2003. The feeding ecology of larval Pacific hake (Merluccius productus) in the California Current region: an updated approach using a combined OPC/ MOCNESS to estimate prey biovolume. Fisheries Oceanography 12: 34-48.

Castro LR, VA Troncoso \& DR Figueroa. 2007. Fine-scale vertical distribution of coastal and offshore copepods in the Golfo de Arauco, central Chile, during the upwelling season. Progress in Oceanography 75: 486-500.

Cornejo R \& R Koppelmann. 2006. Distribution patterns of mesopelagic fishes with special reference to Vincigerria lucetia Garman 1899 (Posichthyidae: Pisces) in the Humboldt Current region off Peru. Marine Biology 149: 1519-1537.

Cortes E. 1997. A critical review of methods of studying fish feeding based on analysis of stomach contents: application to elasmobranch fishes. Canadian Journal of Fisheries and Aquatic Science 54: 726.738.

Gordon JDM, S Nishida \& T Nemoto. 1985. The diet of mesopelagic fish from the Pacific coasts of Hokkaido, Japan. Journal of Oceanographic Society of Japan 41: 8997.

Hyslop EJ. 1980. Stomach content analysis - a review of methods and their application. Journal of Fish Biology 17: 411-429.
Iizuka K \& S Katayama. 2008. Otolith morphology of teleost fishes of Japan. Bulletin of Fisheries Research Agency 25: $1-222$.

John H-C \& C Zelck. 2001. The larval development of Bathylagus greyae (Bathylagidae). Cybium 25(3): 201-208.

Kendall Jr AW, EH Ahlstrom \& HG Moser. 1985. Early life history stages of fishes and their characters. En: Moser HG (ed). Ontogeny and systematics of fishes, pp. 11-22. Allen Press, Lawrence.

Kobyliansky SG. 1990. Two new species of the genus Bathylagichthys Kobyliansky (Bathylagidae: Salmoniformes) from the Southern Hemisphere Subpolar Waters. Journal of Ichthyology 30(5): 21-27.

Lloris D \& J Rucabado. 1985. A new species from Nansenia ( $N$. problematica) (Salmoniformes, Bathylagidae) from the Southeast Atlantic. Copeia 1985: 141-145.

Matallanas J. 1986. Notes ostéologiques sur Nansenia problematica Lloris et Rucabado 1985, avec discussion de son statut générique. Cybium 10(4): 389-394.

Moser HG. 1996. The early stages of fishes in the California Current region. CalCOFI Atlas 33: 1-1006.

Nelson JS. 2006. Fishes of the world, 622 pp. John Wiley \& Sons, New York.

Pequeño G \& J Matallanas. 2003. Bathylagichthys parini (Osmeriformes: Bathylagidae) from Chilean fjords: new morphological data. Cybium 27(3): 242-244.

Richards WJ. 2006. Early stages of Atlantic fishes: an identification guide of western central North Atlantic, 2672 pp. CRC Press, Florida.

Rosenberg P \& S Palma. 2003. Cladóceros de los fiordos y canales patagónicos localizados entre Golfo de Penas y el Estrecho de Magallanes. Investigaciones Marinas 31(1): 15-24.

Uribe F \& F Balbontín. 2005. First description of larvae of Bathylagichthys parini (Pisces, Bathylagidae) from the Southeastern Pacific. Bulletin of Marine Science 77: 201207.

Recibido el 3 de agosto de 2010 y aceptado el 14 de septiembre de 2010

Vol. 46, №1, 2011 